\title{
Diagnosis and management of essential tremor and dystonic tremor
}

\author{
Alexandre Gironell and Jaime Kulisevsky
}

\begin{abstract}
Essential tremor (ET) is the most common adult movement disorder. Traditionally considered as a benign disease, it can cause an important physical and psychosocial disability. Drug treatment for ET remains poor and often unsatisfactory. Current therapeutic strategies for ET are reviewed according to the level of discomfort caused by tremor. For mild tremor, nonpharmacological strategies consist of alcohol and acute pharmacological therapy; for moderate tremor, pharmacological therapies (propranolol, gabapentin, primidone, topiramate, alprazolam and other drugs); and for severe tremor, the role of functional surgery is emphasised (thalamic deep brain stimulation, thalamotomy). The more specific treatment of head tremor with the use of botulinum toxin is also discussed. Several points are discussed to guide the immediate research into this disease in the near future.

Dystonic tremor is a common symptom in dystonia. Diagnostic criteria for dystonic tremor and differential diagnosis with psychogenic tremor and ET are described. Treatment of dystonic tremor matches the treatment of dystonia. In cases of symptomatic dystonic tremor similar to ET, therapeutic strategies would be the same as for ET.
\end{abstract}

Keywords: essential tremor, propranolol, gabapentin, primidone, topiramate, alprazolam, deep brain stimulation

\section{Introduction}

Essential tremor (ET) is not only the most common movement disorder in the elderly but it is also one of the neurological disorders most frequently seen by general neurologists and specialists within neurology [Louis, 2005; Gironell et al. 2001]. Effective treatment options for the disorder are limited (Figure 1). The traditional view of ET as a benign, familial, monosymptomatic disorder is being replaced by one of ET as a disease or family of diseases with aetiological, clinical and pathological heterogeneity [Louis, 2005].

ET is characterised by the presence of postural and kinetic tremor. In classic ET, upper limbs (95\% of patients) and less commonly the head $(34 \%)$, lower limbs $(30 \%)$, voice $(12 \%)$, tongue $(7 \%)$, face $(5 \%)$, and trunk (5\%) exhibit postural or kinetic tremor [Elble, 2006]. It has traditionally been considered a benign condition because of the perception that it does not reduce life expectancy or cause symptoms other than tremor and impaired tandem walking. However, this disorder may cause substantial physical and psychosocial disability. Tremor amplitude increases gradually over time and patients frequently face growing difficulties with writing, drinking, eating, dressing, speaking, and other fine motor tasks.

\section{Diagnosis}

ET is a clinical diagnosis. Criteria for definite and probable ET include abnormal bilateral postural or kinetic tremor of the hands in the absence of other neurological signs. The clinical criteria were comprehensively reviewed by the Consensus Statement of the Movement Disorder Society on Tremor [Deuschl et al. 1998]. Six routine neurophysiological criteria (EMG and accelerometer) have recently been described [Gironell et al. 2004]: (1) rhythmic burst of postural tremor on EMG; (2) tremor frequency superior or equal to $4 \mathrm{~Hz}$; (3) absence of rest tremor or, if present, frequency $1.5 \mathrm{~Hz}$ lower than the postural tremor; (4) absence of tremor latency from rest to postural position; (5) changes of the dominant frequency
Ther Adv Neurol Disord (2009) 2(4) 215-222 DOI: 10.1177/ 1756285609104791 (C) The Author(s), 2009. Reprints and permissions: http://www.sagepub.co.uk/ journalsPermissions.nav

Correspondence to: Jaime Kulisevsky, MD, PhD

Servei de Neurologia, Hospital de Sant Pau, Barcelona, Spain jkulisevskyasantpau.cat

Alexandre Gironell Movement Disorders Unit, Department of Neurology, Sant Pau Hospital, Autonomous University of Barcelona, Catalonia and Ciberned Centro de Enfermedades Neurodegenerativas en Red), Spain 


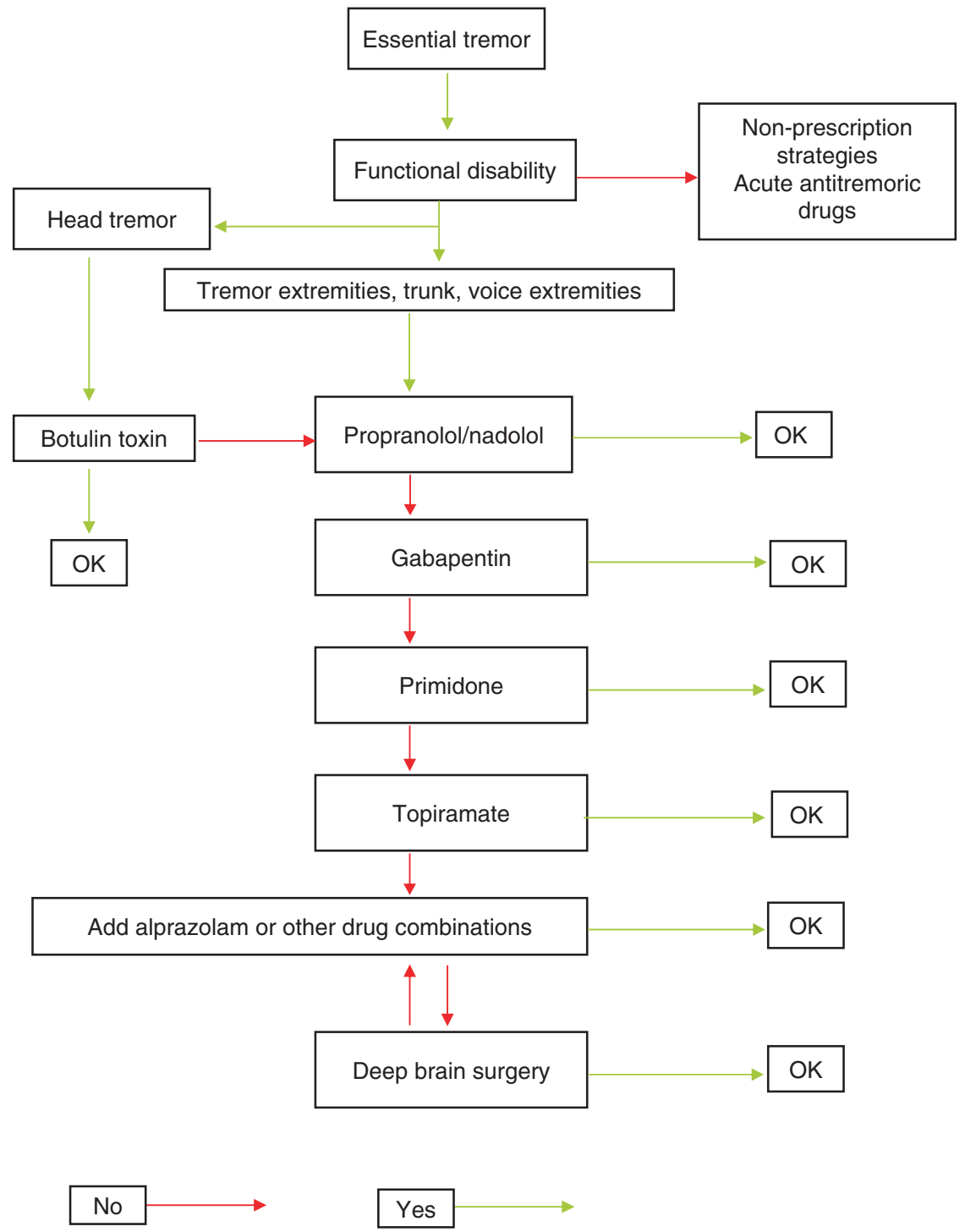

Figure 1. Basic algorithm of current drug treatment in essential tremor.

peak less or equal to $1 \mathrm{~Hz}$ after the weight load test; and (6) no changes in tremor amplitude after mental concentration.

There are several recent excellent reviews on ET treatment [Elble, 2006; Zesiewich et al. 2005]. This paper includes evidence-based medicine observations together with reviewer experience in this field. It is structured according to the severity of patient symptoms.

\section{Management of pauci-symptomatic tremor}

Neuroprotective therapy

There are to date no neuroprotective therapies for ET. A genetic locus will likely be found in the future. With the aid of genetic engineering, it will then be possible to replace the causative mutation into the wild-type allele. This would be a presymptomatic and curative therapy for the disease.

\section{Nonpharmacological strategies}

In milder cases of ET, drug treatment benefits are insufficient to justify continuous chronic treatment. Physicians should explain to patients that psychostimulants such as coffee, tea and cola drinks may have tremoric effects and that anxiety and stress also worsen tremor amplitude. Nonpharmacological anxiolytic measures may be beneficial in milder cases. 


\section{Alcohol}

Ethanol is a potent suppressant of ET in many patients, but it has obvious limitations in the chronic treatment of the disorder. It has been reported that alcohol reduces tremor amplitude in $50-90 \%$ of cases, but tremor may temporarily worsen after the effects of alcohol have worn off [Koller and Biary, 1984]. The mechanism of ethanol in tremor suppression is uncertain. Other alcohols appear to have similar efficacy. Octanol and sodium oxybate were beneficial in pilot studies, but larger double-blind placebocontrolled trials are needed to test the safety, tolerability and efficacy of long-term treatment with these drugs. Sodium oxybate has been approved with restrictions in the United States and several European countries for the treatment of cataplexy [Frucht et al. 2005]. In mild cases of tremor, the physician may suggest an occasional alcoholic beverage (i.e. a beer) approximately 30 minutes prior to a tremor discomfort activity.

\section{Pharmacological therapies}

Another possibility in patients with mild tremor is the use of pharmacological treatment a short time prior to a concrete tremor discomfort activity. The drug should have a rapid absorption and antitremoric action. The most commonly used of such treatments is propranolol (the characteristics of which are discussed later). A dose of 20 $40 \mathrm{mg}$ propranolol about $30-60$ minutes prior to specific actions disrupted by tremor can be used.

\section{Management of moderate tremor}

The patient should be informed that no drug is yet available to entirely eliminate tremor. Antitremoric drugs only ameliorate tremor amplitude. As these drugs may have adverse effects, chronic treatment should be prescribed only if there is some functional disability. Most physicians would agree that drugs should not be prescribed for aesthetic reasons. Common sense is basic in ET treatment. Oral medication commonly used in ET is described in Table 1.

\section{Propranolol}

Propranolol is a nonselective beta-adrenergic receptor antagonist. Twelve controlled studies found it to be efficacious in treating limb tremor in ET (accelerometry was reduced by approximately $50 \%$ ). The dose in these studies ranged from 60 to $320 \mathrm{mg} /$ day. Side effects occurred in $12-66 \%$ of patients and included
Table 1. Oral medication used in the treatment of essential tremor.

\begin{tabular}{llc}
\hline & $\begin{array}{l}\text { Normal } \\
\text { starting } \\
\text { dose } \\
\text { (mg/day) }\end{array}$ & $\begin{array}{l}\text { Normal } \\
\text { therapeutic } \\
\text { dose } \\
\text { (mg/day) }\end{array}$ \\
\hline Propranolol & 10 & $120-320$ \\
Nadolol & 40 & $40-80$ \\
Atenolol & 50 & $50-200$ \\
Primidone & 62.5 & $62.5-100$ \\
Gabapentin & 400 & $1200-3600$ \\
Topiramate & 25 & $200-400$ \\
\hline
\end{tabular}

fatigue, impotence, bradycardia and lightheadedness [Koller and Vetere-Overfield, 1989; Cleeves and Findley, 1988; Jefferson et al. 1979; Tolosa and Loewnson, 1975]. Although propranolol is generally well tolerated, it should be administered with caution in view of contraindications, which include asthma, congestive heart failure, diabetes mellitus and atrioventricular block. Several beta blockers, including sotalol and atenolol, seem to be effective in the management of ET, but propranolol, a nonselective antagonist, has been the most consistently studied and it is more effective that relative selective beta-1 antagonists. Sustained-release propranolol is as effective as conventional propranolol, although patients usually prefer the classical propranolol formulation because its effects are more evident and rapid. Only $25 \%$ of patients maintain their initial good response at 2 years [Koller and Vetere-Overfield, 1989].

In our experience, beta blockers are the best pharmacological treatment for ET and twothirds of patients improve. We usually start with nadolol rather than propranolol because of its more convenient posology (two $40 \mathrm{mg}$ doses per day). As stated above, the major problem with beta blockers is the contraindications, which are very common in older ET patients.

\section{Primidone}

Primidone is an anticonvulsant agent that is metabolised to phylethylmalonamide and phenobabital. Its efficacy in treating ET has been examined in several articles [Chakrabarti and Pearce, 1981; Gorman et al. 1986; Findley et al. 1985; Sasso et al. 1990] in which doses from 50 to 1000 $\mathrm{mg} /$ day were useful. The mean reduction in tremor magnitude by accelerometry was approximately $50 \%$. Primidone has been associated with 
a moderate to high frequency of adverse events that were more severe at treatment initiation. These include sedation, drowsiness, fatigue, nausea, vomiting, ataxia, malaise, dizziness, unsteadiness, confusion, vertigo and an acute toxic reaction. One study showed that slow titration did not improve tolerability to the drug. Another study showed no significant differences in tremor improvement with doses ranging from 250 to $750 \mathrm{mg} /$ day. Prospective, randomised clinical trials indicate that propranolol and primidone reduce limb tremor in ET. Magnitudes of effect of primidone and propranolol were approximately similar [Gorman et al. 1986]. In our experience, the main drawback with primidone is the high prevalence of adverse effects, especially in older patients. For this reason it can be considered a second-line drug, at a similar level to gabapentin.

\section{Gabapentin}

Gabapentin is an anticonvulsant that is structurally similar to the inhibitory neurotransmitter GABA. There is some evidence that there is a disturbance in the GABAergic system in ET. In two of three clinical trials, gabapentin (doses ranging from 1200 to $3600 \mathrm{mg}$ daily) resulted in a significant reduction in tremor compared with placebo, and in one of the two, its effect was similar to that of propranolol [Ondo et al. 2000; Gironell et al. 1999; Pahwa et al. 1998]. Gabapentin is generally well tolerated.

In our experience approximately one-third of patients improve with gabapentin. Its main drawback is this low efficacy rate; however, it is well tolerated and there are very few contraindications. It is a suitable therapy for older patients (the majority of ET patients). If beta blockers are not tolerated or are contraindicated, it is thus a valid option prior to primidone.

\section{Topiramate}

Topiramate is an anticonvulsant that blocks sodium channels and potentiates GABA activity. Several studies, including one double-blind placebo-controlled trial, reported an $18-23 \%$ improvement in clinical rating scales with topiramate use. The dropout rate was approximately $40 \%$ due to appetite suppression, weight loss, paresthesias, anorexia and concentration difficulties [Connor et al. 2008].

In our experience, the efficacy of topiramate is similar to gabapentin, but titration is long and difficult, and tolerance is poor compared to gabapentin. In view of these limitations it is mainly used as a third-line therapy.

\section{Alprazolam}

Alprazolam is a short-acting benzodiazepine. Several studies have found that doses of 0.125 $3 \mathrm{mg} /$ day reduce limb tremor $(25-34 \%)$ as compared with placebo [Huber and Paulson, 1988]. Side effects range from none to $50 \%$ in these studies and include mild sedation and fatigue. Alprazolam is probably efficacious in treating ET, but caution with its use is recommended due to its abuse potential. Alprazolam is usually used as a coadjuvant therapy associated with other drugs in cases of severe tremor.

\section{Other drugs and therapies}

The following agents possibly reduce limb tremor associated with ET.

- Clonazepam significantly reduced kinetic tremor in a study with 14 patients who received doses ranging from 0.5 to $6 \mathrm{mg} /$ day. In another study efficacy was low and drowsiness was a frequent adverse effect. Caution is advised with the use of clonazepam due to its abuse potential and possible withdrawal symptoms following abrupt discontinuance. We use clonazepam preferentially in cases of head, voice and trunk tremor as a coadjuvant therapy [Thomson et al. 1984].

- Clozapine is an atypical neuroleptic with minimal extrapyramidal side effects. Two studies have found that clozapine reduces ET in doses of $6-75 \mathrm{mg} /$ day. In one study $87 \%$ of patients had at least a $50 \%$ reduction in tremor. The major danger is agranulocytosis [Ceravolo et al. 1999].

- At a dose of $30 \mathrm{mg}$ four times daily, nimodipine (a calcium channel blocker) reduced tremor amplitude by $53 \%$ as measured by accelerometry in one study [Biary et al. 1995].

- Botulinum toxin injection in forearm muscles produces little if any improvement in most patients, and finger or wrist weakness is a common side effect [Jankovic et al. 1996].

- In a crossover study, which included ten patients with ET, a single session of $1 \mathrm{~Hz}$ repetitive transcranial magnetic stimulation (rTMS) given over the cerebellar vermis was compared with a sham rTMS condition. The study found improvement with a standard tremor scale and accelerometry at $5 \mathrm{~min}$ after rTMS, but not after sham stimulation. No difference between real and sham stimulation was seen at $60 \mathrm{~min}$ [Gironell et al. 2002]. 
- A single dose of $1000 \mathrm{mg}$ levetiracetam in a double-blind controlled study found a significant reduction in tremor amplitude after 2 hours using accelerometry and functional tests. However, two further controlled studies have not revealed any antitremoric action of levetiracetam compared with placebo [Elble et al. 2007].

- A pilot study with zonisamide $(200 \mathrm{mg} /$ day $)$ showed antitremoric efficacy in accelerometry but not in clinical and functional scales. Another open-label trial showed modest improvements in tremor scores [Ondo, 2007].

- A single pilot, double-blind, placebo-controlled trail with pregabalin revealed significant improvements in tremor clinical scores [Zesiewicz et al. 2007].

\section{Management of head and voice tremor}

Pharmacologic management of essential head and voice tremor is less efficient than that of hand tremor. Propranolol and primidone, alone or in combination, have been recommended for essential head tremor. Clonazepam is often recommended, but controlled studies are not available. A promising therapy for head tremor is the local injection of botulinum toxin, but further studies are needed to confirm this [Pahwa et al. 1995]. Deep brain stimulation is also effective for head and voice tremor, but bilateral ventralis intermedius (VIM) stimulation is usually necessary. In our experience, the response to botulinum toxin is good in head tremor and we usually prefer this treatment before other oral pharmacological therapies in patients with predominant moderate and severe head tremor.

\section{Management of severe tremor}

\section{Pharmacological therapy}

This is of limited aid in cases of severe tremor, especially when beta blockers cannot be used. In these cases, we usually propose surgery, especially in patients less than 70 years old. The age is a relative criterion. In patients aged 70-75 years, unilateral surgery may be performed to minimise the risk of pseudobulbar and cognitive sequelae. Patients with severe tremor are usually treated with polytherapy that includes a combination of beta blockers, primidone, gabapentin or topiramate, and benzodiazepines.

\section{Surgery}

VIM nucleus thalamotomy and deep brain stimulation (DBS) produce marked ( $>75 \%)$ or complete suppression of limb tremor in $70-90 \%$ of patients. Surgery is therefore the most effective treatment for ET [Vaillancourt et al. 2003]. Thalamotomy and DBS are reserved for drugresistant tremor that warrants surgery. DBS is the procedure of choice at most centres because it is associated with fewer adverse events than thalamotomy, particularly when bilateral procedures are performed. In a multicentre European study in which 37 patients with ET were followed for a mean of 6.5 years, VIM DBS offered longterm benefits and safety [Sydow et al. 2003].

VIM is the preferred stereotactic target in nearly all patients with ET. However, alternative targets may be needed in some situations. Parkinson tremor and ET in the same patient have responded to subthalamic DBS. Targets other than VIM should therefore be investigated in patients who are refractory to VIM DBS.

Several uncontrolled studies have reported favourable results with gamma knife thalamotomy. However, delayed complications have been observed and clinical improvement may take weeks to months [Young et al. 2000]. Controlled studies of gamma knife thalamotomy should be considered in advanced patients who are not candidates to surgery. DBS achieves excellent results in ET patients, and total suppression of antitremoric medication may be possible. There is a general consensus among experts that better results are obtained in postural ET tremor than in task-specific tremor.

\section{Future research endpoints}

Although it is the most frequent movement disorder, research in ET treatment is still scarce. The therapeutic arsenal and efficacy rates of antitremoric drugs are very limited. In the near future, ET research must be focused as follows:

(1) To study the physiopathology of ET, to determine the neurotransmitter system or systems involved. To correlate these findings with the clinical heterogeneity of the disease. These studies would permit the understanding the variation of response to treatment in patients with ET and would guide the clinician in the selection of appropriate medication for each patient. 
(2) Cost versus benefit studies of ET treatments.

(3) Development of neuroprotective therapies.

(4) Genetic studies trying to identify the genetic loci related to familial ET.

(5) Controlled studies on pharmacological and surgical treatment of head and vocal tremor.

(6) Prospective, placebo-controlled, doubleblind and multicentre studies to determine the efficacy and the adverse effects of pharmacological and surgical treatments in ET.

Diagnosis and management of dystonic tremor It is well known that dystonic patients sometime have rhythmic movements, particularly in the arms and neck, manifested as tremor [Jankovic and Fahn, 1980]. In one survey, $68 \%$ of patients with cervical dystonia had head tremor [Pal et al. 2000]. Two basic types of tremors are seen in dystonic patients: an accompanying postural/ action tremor that resembles ET, or an enhanced physiologic tremor and a tremor that is a rhythmic expression of rapid dystonic movements. The latter can usually be distinguished from the former by showing that the tremor appears only when the affected body part is placed in a position of opposition to the major direction of pulling by the abnormal dystonic contractions and disappears when the body part is positioned where the dystonia wants to place it.

\section{Diagnosis}

The clinical criteria of dystonic tremor syndromes were reviewed by the Consensus Statement of the Movement Disorder Society on Tremor [Deuschl et al. 1998]. They propose three possible definitions:

(1) Dystonic tremor: tremor in a body part affected by dystonia. This involves: tremor in an extremity or body part that is affected by dystonia, focal tremors, usually with irregular amplitudes and variable frequency (mainly less than $7 \mathrm{~Hz}$ ), and mainly postural/kinetic tremors usually not seen during complete rest.

(2) Tremor associated with dystonia: this tremor occurs in a body part not affected by dystonia, but the patient has dystonia elsewhere.

(3) Dystonia gene-associated tremor: tremor as an isolated finding in patients with a dystonic pedigree.

(4) Dystonic tremor appears to be less regular than ET [Jedynak et al. 1991]. Sometimes, it is very difficult to distinguish between the two types, particularly with writing tremor and cervical tremor. Primary writing tremor can sometimes represent a task-specific dystonia or task-specific ET [Elble et al. 1990]. A family history of tremor is increased in dystonic patients [Fletcher et al. 1991]. Although accompanying ET is recognised in patients with dystonia, there is uncertainty as to how common this occurrence is. Tremor of the hands can be seen fairly often in patients with cervical dystonia.

Differentiation of psychogenic from dystonic tremor can be difficult [McAuley and Rothwell, 2004]. In fact, in dystonic tremor duration of tremor burst is widely variable reflecting its jerky nature and similar to some psychogenic tremors. Conversely, psychogenic tremor may have selective tremor absence and lack of disability, whereas dystonic tremor has selective disability and is usually steroeotypic.

\section{Treatment}

Tremor is one of the clinical symptoms of dystonia. The treatment of dystonic tremor matches the treatment of dystonia. There are no specific therapeutic trials evaluating treatment efficacy on dystonic tremor. In cases of postural or intention tremor very similar, and difficult to discriminate from ET, therapeutic tremor strategies would be the same as in ET patients.

\section{Conflict of interest statement}

None declared.

\section{References}

Biary, N., Bahou, Y., Sofi, M.A., Thomas, W. and Al Deeb, S.M. (1995) The effect of nimodipine on essential tremor. Neurology 45: 1523-1525.

Ceravolo, R., Salvetti, S., Piccini, P., Lucetti, C., Gambaccini, G. and Bonuccelli, U. (1999) Acute and chronic effects of clozapine in essential termor. Mov Disord 14: 468-472.

Chakrabarti, A. and Pearce, J.M.S. (1981) Essential tremor: response to primidone. $\mathcal{F}$ Neurol Neurosurg Psychiatry 44: 650.

Cleeves, L. and Findley, L.J. (1988) Propranolol and propranolol-LA in essential tremor: a double blind comparative study. $\mathcal{F}$ Neurol Neurosurg Psychiatry 51: 379-384.

Connor, G.S., Edwuards, K. and Tarsy, D. (2008) Topiramate in essential tremor: findings from double-blind, placebo-controlled, crossover trials. Clin Neuropharmacol 31: 97-103.

Deuschl, G., Bain, P., Brin, M. and an Ad Hoc Scientific Committee (1998) Consensus statement 
of the Movement Disorder Society on tremor, Mov Disord 13(Suppl. 3): 2-23.

Elble, R.J., Moody, C. and Higgins, C. (1990) Primary writing tremor: a from of focal dytonia. Mov Disord 5: $118-126$.

Elble, R.J. for the Tremor Research Group and conference attendees (2006) Report from a U.S. Conference on essential tremor. Mov Disord 21: 2052-2061.

Elble, R.J., Lyons, K.E. and Pahwa, R. (2007) Levetiracetam is not effective for essential tremor. Clin Neuropharmacol 30: 350-356.

Findley, L.H., Cleeves, L. and Calzetti, S. (1985) Primidone in essential tremor of the hands and head: a double blind controlled clinical study. f Neurol Neurosurg Psychiatry 48: 911-915.

Fletcher, N.A., Harding, A.E. and Marsden, C.D. (1991) Intrafamilial correlation in idopathic torsion dystonia. Mov Disord 6: 310-314.

Frucht, S.J., Houghton, W.C., Bordelon, Y., Greene, P.E. and Louis, E.D. (2005) A single-blind, open-label trial of sodium oxybate for myoclonus and essential tremor. Neurology 65: 1967-1969.

Gironell, A., Kulisevsky, J., Barbanoj, M., LópezVillegas, D., Hernández, G. and Pascual-Sedano, B. (1999) A double-blind placebo-controlled trial of gabapentin and propranolol in patients with essential tremor. Arch Neurol 56: 475-480.

Gironell, A., Kulisevsky, J., Barbanoj, M., Gich, I., Pascual-Sedano, B. and Otermín, P. (2001) Temblor postural: estudio clínico y neurofisiológico en una serie consecutiva de 300 pacientes. Med Clin (Barc) 117: 601-605.

Gironell, A., Kulisevsky, J., Lorenzo, J., Barbanoj, M., Pascual-Sedano, B. and Otermín, P. (2002)

Transcranial magnetic stimulation of the cerebellum in essential tremor: a controlled study. Arch Neurol 59: 413-417.

Gironell, A., Kulisevsky, J., Pascual-Sedano, B. and Barbanoj, M. (2004) Routine neurophysiological tremor analysis as a diagnostic tool for essential tremor: a prospective study. $\mathcal{F}$ Clin Neurophysiol 21: $446-450$.

Gorman, W.P., Cooper, R., Pocock, P. and Campbell, M.J. (1986) A comparison of primidone, propranolol, and placebo in essential tremor, using quantitative analysis. $\mathcal{F}$ Neurol Neurosurg Psychiatry 49: 64-68.

Huber, S.J. and Paulson, G.W. (1988) Efficacy of alprazolam for essential tremor. Neurology 38: 241-243.

Jankovic, J. and Fahn, S. (1980) Physiologic and pathologic tremor. Diagnosis, mechanisms, and management. Ann Int Med 93: 460-465.

Jankovic, J., Schwartz, K., Clemence, W., Aswad, A. and Mordunt, J. (1996) A randomized, double-blind, placebo-controlled study to evaluate botulinum toxin type A in essential hand tremor. Mov Disord 11: 250-256.

Jedynak, C.P., Bonnet, A.M. and Agid, Y. (1991)

Tremor and idiopathic dystonia. Mov Disord 6:

230-236.

Jefferson, D., Jenner, P. and Marsden, C.D. (1979)

B-adrenoreceptor antagonists in essential tremor.

f Neurol Neurosurg Psychiatry 41: 904-909.

Koller, W.C. and Biary, N. (1984) Effect of alcohol on tremors: comparison with propranolol. Neurology 34: 221-222.

Koller, W.C. and Vetere-Overfield, B. (1989)

Acute and chronic effects of propranolol and primidone in essential tremor. Neurology 39: 1587-1588.

Louis, E.D. (2005) Essential tremor. Lancet Neurol 4: $100-110$.

McAuley, J. and Rothwell, J. (2004) Identification of psychogenic, dystonic, and other organic tremor by a coherence entrainment test. Mov Disord 19: 253-267.

Ondo, W., Hunter, C., Dat Wuong, K., Scwartz, K. and Jankovic, J. (2000) Gabapentin for essential tremor: a multiple-dose, double-blind placebocontrolled trial. Mov Disord 15: 678-682.

Ondo, W.G. (2007) Zonisamide for essential tremor. Clin Neuropharmacol 30: 345-349.

Pal, P.K., Samii, A., Schulzer, M., Mak, E. and Tsui, J.K. (2000) Head tremor in cervical dystonia. Can F Neurol Sci 27: 137-142.

Pahwa, R., Busenvark, K., Swanson-Hyland, E.F., Dubinsky, R.M., Hubble, J.P., Gray, C. and Koller, W.C. (1995) Botulinum toxin treatment of essential head tremor. Neurology 45: $822-824$

Pahwa, R., Lyons, K., Hubble, J.P., Busenbark, K., Rienerth, J.D., Pahwa, A. and Koller, W.C. (1998) Double-blind controlled trial of gabapentin in essential tremor. Mov Disord 13: 465-467.

Sasso, E., Perucca, E., Fava, R. and Clalzetti, S. (1990) Primidone in the long-term treatment of essential tremor: a prospective study with computerized quantitative analysis. Clin Neuropharmacol 13: 67-76.

Sydow, O., Thobois, S., Alesch, F. and Speelman, J.D. (2003) Multicentre European study of thalamic stimulation in essential tremor: a six year follow up. f Neurol Neurosurg Psychiatry 74: 1387-1391.

Thomson, C.M., Lang, A., Parkes, J.D. and Marsden, C.D. (1984) A double-blind trial of clonazepam in benign essential tremor. Clin Neuropharmacol 7: 83-88.

Tolosa, E.S. and Loewnson, R.B. (1975) Essential tremor: treatment with propranolol. Neurology 25: 1041-1044. 
Visit SAGE journals online http://tan.sagepub.com

@SAGEJOURNALS

Online
Vaillancourt, D.E., Sturman, M.M., Verhangen

Metman, L., Bakay, R.A. and Corcos, D.M. (2003)

Deep brain stimulation of the VIM thalamic nucleus modifies several features of essential tremor. Neurology 61: 919-925.

Young, R.F., Jacques, K., Mark, R., Kopyov, O., Copcutt, B., Posewitz, A. et al. (2000) Gamma knife thalamotomy for treatment of tremor: long-term results. $\mathcal{F}$ Neurosurg 93: $128-135$.
Zesiewich, T.A., Elble, R., Louis, M.D., Hauser, R.A., Sullivan, K.L., Dewey, R.B. et al. (2005) Practice parameter: therapies for essential tremor. Report of the quality standards subcommittee of the American Academy of Neurology. Neurology 64: 2008-2020.

Zesiewicz, T.A., Ward, C.L., Hauser, R.A., Salemi, J.L., Siraj, S., Wilson, M.C. et al. (2007) A pilot, double-blind, placebo-controlled trial of pregabalin in the treatment of essential tremor. Mov Disord 22: $1660-1663$. 